\title{
Cooperative Selection: Improving Tournament Selection via Altruism
}

\author{
Juan Luis Jiménez Laredo ${ }^{1}$, Sune S. Nielsen ${ }^{1}$, Grégoire Danoy ${ }^{1}$, \\ Pascal Bouvry ${ }^{1}$, and Carlos M. Fernandes ${ }^{2}$ \\ 1 FSTC-CSC/SnT, \\ University of Luxembourg, 6, rue Richard Coudenhove-Kalergi, \\ L-1359 Luxembourg, Luxembourg \\ e-mail: \{juan.jimenez, sune.nielsen, gregoire.danoy, pascal.bouvry\}@uni.lu \\ ${ }^{2}$ Laseeb: Evolutionary Systems and Biomedical Engineering, \\ Technical University of Lisbon, Lisbon, Portugal \\ e-mail: cfernandes@laseeb.org
}

\begin{abstract}
This paper analyzes the dynamics of a new selection scheme based on altruistic cooperation between individuals. The scheme, which we refer to as cooperative selection, extends from tournament selection and imposes a stringent restriction on the mating chances of an individual during its lifespan: winning a tournament entails a depreciation of its fitness value. We show that altruism minimizes the loss of genetic diversity while increasing the selection frequency of the fittest individuals. An additional contribution of this paper is the formulation of a new combinatorial problem for maximizing the similarity of proteins based on their secondary structure. We conduct experiments on this problem in order to validate cooperative selection. The new selection scheme outperforms tournament selection for any setting of the parameters and is the best trade-off, maximizing genetic diversity and minimizing computational efforts.
\end{abstract}

\section{Introduction}

Evolutionary algorithms (EAs) are optimization meta-heuristics inspired by the Darwinian process of natural selection. As in nature, individuals in EAs compete for survival and the fittest are preferentially selected for reproduction. Through the course of generations, evolution operates bottom up by filtering good genes in the population and optimizing the design of the individuals. This complex process is triggered by a set of simple rules, the cornerstones being the breeding operators and the selection scheme: the formers leveraging the proper mixing of individuals' structures and the latter balancing the selection pressure. If the selection pressure is too intensive, the population will lose genetic diversity quickly and the algorithm will converge to local optima. On the other hand, if the selection pressure is too relaxed, the speed of convergence will slow down and the algorithm will waste computational efforts. Hence, the design of efficient selection schemes remains as an open topic of research in Evolutionary Computation. 
In addition to canonical approaches such as ranking or roulette wheel [4], other selection schemes have been designed, e.g. to balance exploration and exploitation [1], to autonomously decide on the state of reproduction of individuals [7], or to be able to self-adapt the selection pressure on-line [3]. Nevertheless, tournament selection [8] is still one of the most studied and employed schemes for selection. Tournament selection is an easy-to-implement easy-to-model scheme, in which the best of $t$ randomly sampled individuals is selected for mating. This simple scheme has many advantages such as allowing the tuning of the selection pressure by simply varying the size of the tournament, having a low algorithmic complexity (i.e. $O(t)$ for a single selection) and being suitable for parallel and decentralized execution as it does not require to compute estimates of the global population. However, tournament selection has been also reported to have some disadvantages that we aim to cover in this paper.

Poli [9] points out two different phases in a tournament: the sampling phase where $t$ individuals are randomly chosen from the population, and the selection phase, in which the best of the sampled individuals is selected for reproduction. The two main issues arising from Poli's study are known as the not-sampling and multi-selection problems, both of them responsible for the loss of diversity. The not-sampling issue refers to the probability of an individual for not being sampled. Xie, Zhang and Andreae [14] conduct a thorough analysis on this issue and conclude that, independently of the population size, some individuals in every generation will never be sampled when using small tournament sizes, e.g. for binary tournament $13 \%$ of the individuals are never sampled, if $t=3$ that value is $\sim 5 \%$, and so on. In contrast, the multi-selection ${ }^{3}$ issue refers to the probability of an individual for being selected multiple times in the selection phase. The risk here is that, if the tournament size is large, a small elite of individuals could take over the population and quickly reduce the diversity. Given that the only tunable parameter in tournament selection is the tournament size, either the not-sampling or multi-selection issue will always be present to some extent at any value of the parameter.

Some authors have presented alternative solutions to the not-sampling problem. The backward-chaining proposal by Poli [9], the unbiased tournament selection by Sokolov and Whitley [12] or the round-replacement by Xie and Zhang [13] successfully address the issue. In their respective proposals, all authors are able to save the computational efforts associated to the evaluation of not-sampled individuals. Xie and Zhang, however, show that the sampling countermeasures are only effective in the case of binary tournament. For larger tournament sizes they claim that "the not sampled issue in standard tournament selection is not critical". On the basis of previous findings the authors recommend that research should focus on tuning the parent selection pressure instead of developing alternative sampling replacement strategies.

\footnotetext{
${ }^{3}$ The multi-selection problem is alternatively referred to as not-selection problem, i.e. the multiple selection of some individuals implies the opposite problem, some individuals are never selected.
} 
With such a recommendation in mind, cooperative selection [6] tackles the selection phase in tournament selection. The aim is to minimize the loss of diversity due to the multi-selection of elite individuals. Given that the multi-selection issue is more relevant for large tournament sizes, the not-sampling of individuals is assumed as a minor issue. Nonetheless, any of the aforementioned not-sampling solutions could be easily integrated within our approach as they are complementary. Cooperative selection tries to prevent the multi-selection of elite individuals by implementing an altruistic rule: after winning a tournament, an individual must decrease its own fitness as to yield its position to the second-ranked individual for future tournaments.

In this paper, we analyze first the properties of the approach under simplified assumptions. Then, in order to validate such properties in a real problem, we tackle a new combinatorial problem designed to maximize the similarity between proteins. In that context, cooperative selection is shown to preserve a higher genetic diversity than tournament selection and to require less evaluations to yield the same quality in solutions.

The rest of the paper is structured as follows. Section 2 introduces the new selection scheme. This section also analyzes the main properties of the approach and provides some assessment for tuning the scheme parameters. Section 3 presents a new combinatorial problem that is used afterwards to validate the cooperative selection scheme. Finally, some conclusions and future lines of research are exposed in section 4 .

\section{Cooperative Selection}

Cooperative selection is a variant in the selection phase of tournament selection. This section presents a description of the new selection scheme and analyzes its properties for different parameter values using tournament selection as the counterpart for comparison.

\subsection{Scheme description}

In order to describe cooperative selection, let us first introduce the following definitions and nomenclature:

- An association $\vec{a}=\left\{\overrightarrow{x_{1}}, \ldots, \overrightarrow{x_{a}}\right\}$ is the scheme's equivalent of a tournament, where a set of individuals $\overrightarrow{x_{i}}: i \in\{1, \ldots, a\}$ is randomly sampled from a population $P$ to compete for selection in an association of size $a$.

- Every individual $\vec{x}=\left\{x_{1}, \ldots, x_{l}\right\} \mid \vec{x} \in P$ has a length of $l$ parameters and is characterized by two different fitness metrics:

- The canonical fitness function $f(\vec{x})$.

- A transcription of $f(\vec{x})$ called cooperative fitness $f_{\text {coop }}(\vec{x})$, which is initialized in an atomic operation:

$$
\text { sync.eval }(\vec{x})\left\{f(\vec{x}), f_{\text {coop }}(\vec{x}) \leftarrow f(\vec{x})\right\}
$$

Hence, $f_{\text {coop }}(\vec{x})=f(\vec{x})$ holds true right after the evaluation of $\vec{x}$. 
Given an association $\vec{a}$ as provided by the sampling method described above, Procedure 1 details the criteria to select an individual from $\vec{a}$. Note here that $\vec{a}$ and an analogue tournament $\vec{t}$ are indistinguishable and therefore, the only difference between cooperative and tournament selection relies on this procedure.

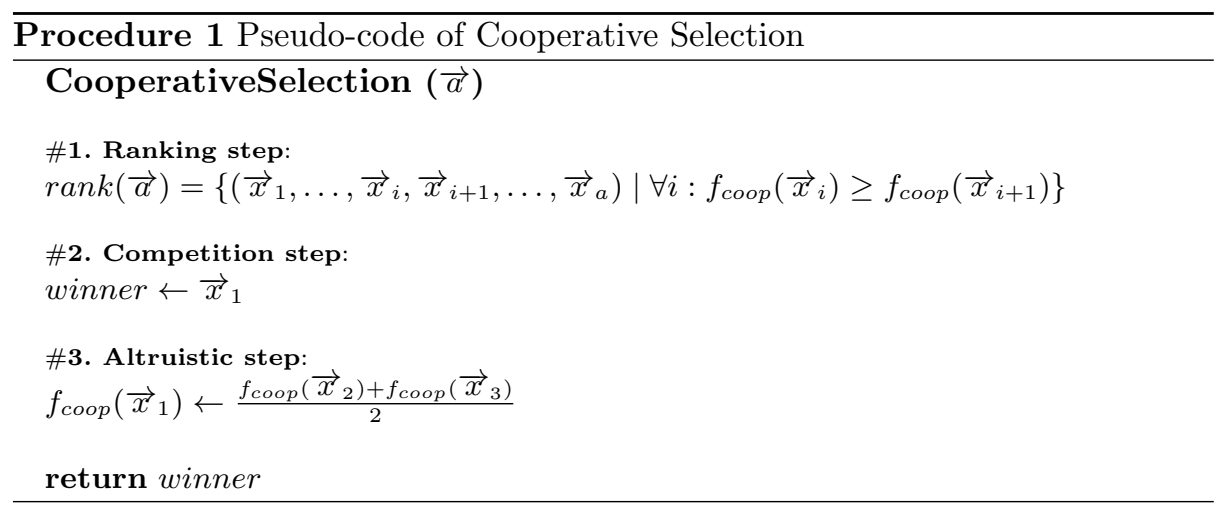

The competition (or selection phase) in cooperative selection consists of three steps. In the first one, the sampled individuals $(\vec{a})$ are ranked according to their cooperative fitness. To that end, without any loss of generality, we have considered in this paper the case of maximizing the fitness. In a second step, the individual with the highest cooperative fitness $f_{\text {coop }}\left(\vec{x}_{1}\right)$ is selected as the winner of the competition. However, these two steps would not change from a canonical competition if it were not for the final step where, after being selected, the winner altruistically decreases its own cooperative fitness. In the next section we show that such a simplistic rule has a tremendous impact on reducing the environmental selection pressure and consequently the loss of genetic diversity.

\subsection{Properties and tuning of parameters}

Cooperative selection is based on tournament selection and, as in tournament selection, the scheme can also be divided into two main phases: the sampling phase in which the individuals are randomly sampled to create an association, and the selection phase in which the individual with the highest cooperative fitness is selected for reproduction. In order to analyze the properties of cooperative selection, this section tackles both phases incrementally by assessing first the bias introduced during the sampling phase, and then by analyzing the combined effect of the not-sampling and not-selection of individuals during the selection phase.

The sampling phase. The sampling of individuals in cooperative selection can be described by the probability model presented by Xie et al. [14] for tournament 
selection. The model states that the probability of an individual to be sampled in $y \in\{1, \ldots, P\}$ associations/tournaments is:

$$
1-\left(1-\frac{1}{P}\right)^{y a}
$$

where $P$ is the population size, $a$ the association/tournament size, and $y$ the number of associations/tournaments within the generation.
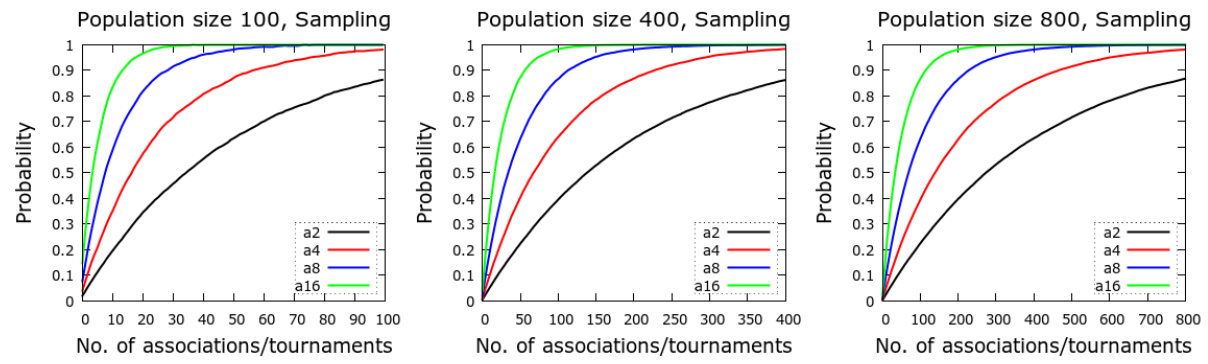

Fig. 1. Probability of sampling an individual at least once per generation for $y=P$. Given that the sampling of individuals is performed identically in tournament and cooperative selection, these results hold in both schemes. Results are the average of 30 independent runs.

In Fig. 1 we verify empirically the model via simulations and estimate the sampling probabilities for $P \in\{100,400,800\}$ and $a \in\{2,4,8,16\}$. The obtained results show that the association size $(a)$ is the main factor influencing the sampling probability while the population size $(P)$ does not seem to affect the probability trend (Sokolov and Whitley [12] show that the population size may indeed affect the trend if $P<10$ however populations in EAs are usually not that small). Independently of the population size, there is a certain probability for an individual of not being sampled when $a$ is small. An individual that is not sampled does not have any chance to pass to subsequent generations and thus, its associated genetic material and computational efforts are hopelessly wasted. Such a waste amounts to $\sim 13 \%$ of the population for $a=2$; a percentage that becomes progressively smaller as the association size increases and that can be neglected from $a>5$. Therefore, the association size should be preferentially set to large values for an optimal operation of the scheme.

The selection phase. Once an association $\vec{a}=\left\{\overrightarrow{x_{1}}, \ldots, \overrightarrow{x_{a}}\right\}$ is sampled from the population, the selection phase is responsible for determining which individual in $\vec{a}$ will be finally selected for reproduction. At this stage, a large association size entails an increase in the selection pressure and a loss of genetic diversity. Such an effect is well known in tournament selection for large tournament sizes and needs to be assessed for cooperative selection. 
Xie and Zhang [13] propose an experimental method for assessing the loss of diversity according to different values of $a$. The method employs a synthetic population called fitness rank distribution (FRD). The idea behind a FRD is that a population can be partitioned into different bags of individuals with an equal fitness. Given that each of these bags has an associated fitness rank, a selection scheme can be characterized by the amount of individuals which are selected from the different bags. In order to analyze cooperative selection, we will assume a simplistic scenario in which the FRDs emulate the fitnesses of a randomly initialized population in an easy problem [11]. Fig. 2 shows three of these FRDs in which the best individuals are ranked first.
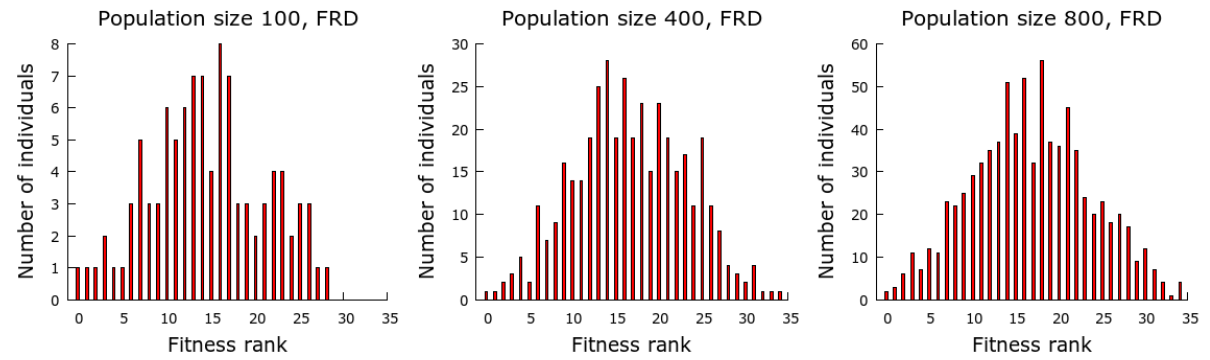

Fig. 2. Fitness rank distributions for three different population sizes. FRDs are dropped from a normally distributed variable $X \sim \mathcal{N}(0,36)$ and then shifted until $\min (X)=$ 0 . This method approximates a $\mathcal{N}(18,36)$ distribution and guarantees non-negative values.

Using a FRD as an input, our genetic algorithm assumes a generational scheme with selection as the single operator to be applied. Any possible loss of diversity is therefore due to the selection scheme and can be assessed by computing the amount of individuals discarded from one generation to the other. In this context, there are two possible causes for losing diversity: those individuals that are not-sampled, and those ones that being sampled are not-selected.

Fig. 3 compares the loss of diversity in tournament and cooperative selection as a function of the tournament/association size. The selection pressure monotonically increases for both schemes. However, cooperative selection scales more gracefully than tournament selection: for $a=t=20$ cooperative selection outperforms tournament selection by a $\sim 24 \%$.

Given the different responses of both schemes to parametrization, we define as analogues a tournament $(t)$ and an association $(a)$ inducing the same loss of diversity, e.g. $t=2$ and $a=8$ in Fig. 3 . This notion is relevant to make comparisons between schemes: since two analogue parameters preserve the diversity equally, one scheme will outperform the other if, for an analogue parametrization, the scheme preferentially selects fitter individuals. Note here that the variety and quality of the selected individuals are two conflicting objectives. 


\section{Tournament selection}

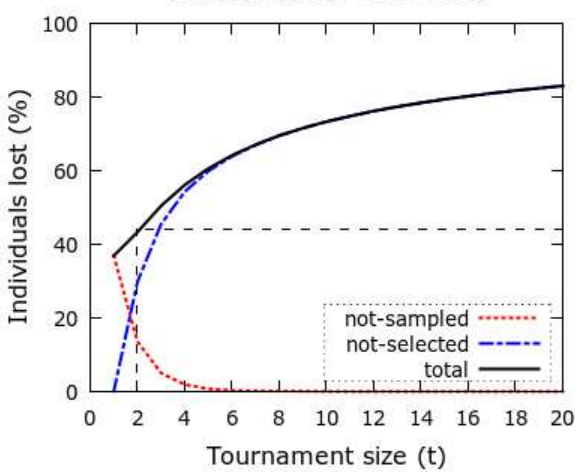

Cooperative selection

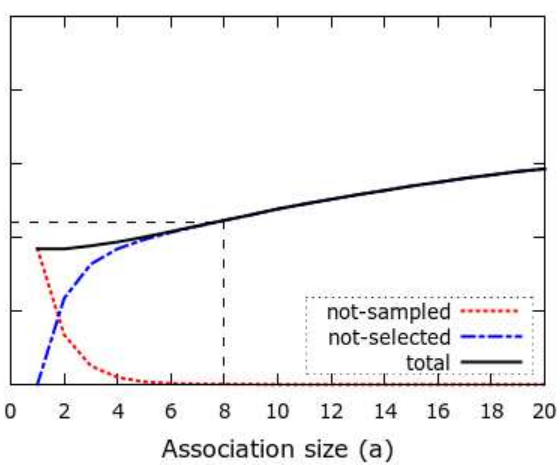

Fig. 3. Loss of diversity in tournament selection (left) and cooperative selection (right) for $P=400$. The dashed line exemplifies an equivalence between analogue tournament and association sizes.

To gain insights into this question, Fig. 4 computes the selection frequencies for different parametrizations of tournament and cooperative selection. The results show two main outcomes:

1. Cooperative selection is more robust to parameter tuning than tournament selection as the size of an association has less impact on the selection frequencies than the respective tournament size.

2. In the case of an analogue parametrization (i.e. $t 2$ is analogue to $a 8$ ), the frequency of good individuals is higher in cooperative selection than in tournament selection.
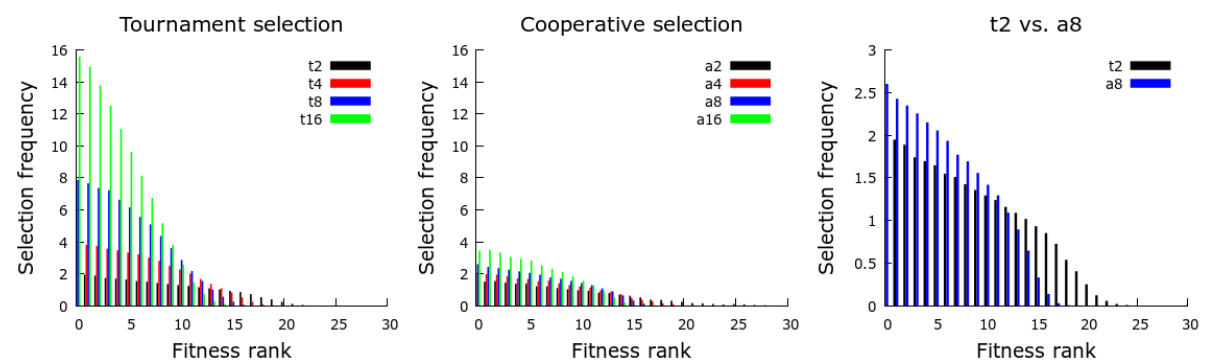

Fig. 4. Expected selection frequencies for different tournament and association sizes in tournament selection (left) and cooperative selection (center). The graph on the right compares the selection frequencies between binary tournament $(t 2)$ and its analogue association size (a8). Frequencies are obtained from 30 independent runs. 


\section{Validating cooperative selection in a noisy real problem}

In order to validate the previous outcomes on cooperative selection, we tackle in this section the problem of finding two proteins (provided as two sequences of codons) with a similar secondary structure. The pairwise comparison of the secondary structure is a multi-modal problem with tools such as the Vector Alignment Search Tool (VAST) ${ }^{4}$ dedicated to classify similarities between proteins. VAST requires a $\mathrm{pdb}^{5}$ file as an input to query a database for similarities. However, pdb's are obtained in laboratories using expensive and time-consuming techniques such as X-ray crystallography or nuclear magnetic resonance [2]. These techniques prevent a systematic search of all possible sequences. In this context, we propose ESSS as a meta-model for exploring the problem landscape.

\subsection{Estimated Secondary Structure Similarity (ESSS)}

The secondary structure is a primary approach to determine the three-dimensional form of an amino acid sequence. Given that the biological functionality of a protein is conferred by its 3D structure, establishing the 3D similarity between two proteins provides an estimation of their related properties. ESSS is an assessment on the similarity of the secondary structure between two sequences.

Using the tool PROFphd [10], the likely secondary structure type $T\left(c_{i}\right)$ can be estimated per codon $c_{i}$ with a reliability $R_{T}\left(c_{i}\right) \in\{1 \ldots 10\}$ in the sequence $C=\left\{c_{1} \ldots c_{N}\right\}$. With $T_{\text {ref }}(i)$ the actual type found at position $i$ of the reference structure, the estimated secondary structure similarity score $F$ is calculated as follows:

$$
F\left(C, T_{\text {ref }}\right)=\sum_{i=1}^{N} \operatorname{Match}\left(T\left(c_{i}\right), T_{r e f}(i)\right)
$$

where

$$
\operatorname{Match}\left(T\left(c_{i}\right), T_{\text {ref }}(i)\right)=\left\{\begin{aligned}
0 & \text { if } T\left(c_{i}\right) \wedge T_{\text {ref }}(i) \notin\{\text { Helix, Sheet }\} \\
R_{T}\left(c_{i}\right) & \text { if } T\left(c_{i}\right)=T_{\text {ref }}(i) \\
-R_{T}\left(c_{i}\right) & \text { if } T\left(c_{i}\right) \neq T_{\text {ref }}(i)
\end{aligned}\right.
$$

The reference types $T_{r e f}(i)$ are extracted from the original pdb file. In this paper, we conduct an experiment using the $256 b$ sequence $^{4}$ as a reference $\left(T_{\text {ref }}\right)$. The $256 b$ sequence is composed of 106 codons and codifies the cytochrome B562 molecule.

Multi-modality. ESSS is a multi-modal problem. Only for the $256 b$ sequence that we tackle in this paper, VAST provides a list of 1635 related structures from which $4 J E A$ is the most similar. $4 J E A$ codifies the soluble cytochrome B562 and differs in only 13 out of 106 codons.

\footnotetext{
4 VAST and pdb's of sequences are available on-line at http://www.ncbi.nlm.nih. gov/Structure/

5 Protein Data Bank format
} 
Noisiness. ESSS theoretical optimum is at $F(256 b, 256 b)=860$ for the instance under study. However, to yield such a value, PROFphd should return the maximum reliability $R_{T}=10$ at any of the 86 positions of the sequence folding as a helix. Given that PROFphd is a meta-model based on a neural network, there is a certain error due to the training process which translates in the actual fitness being $F(256 b, 256 b)=463$. The noisiness of the function can be further proven since the sequence $4 J E A$ scores $F(4 J E A, 256 b)=520$; more than the reference sequence itself, i.e. $F(4 J E A, 256 b)>F(256 b, 256 b)$.

\section{$3.2 \quad$ Experimental setup}

Experiments in this paper are conducted for a generational 1-elitism genetic algorithm. Two versions of the algorithm are considered: one using cooperative selection and the other being tournament selection. In order to validate the properties described in section 2.2, analogue parameters $t=2$ and $a=8$ are tested. Additionally, $t=8$ is also analyzed to check differences with respect to $a=8$. Table 1 presents a summary of all parameter settings.

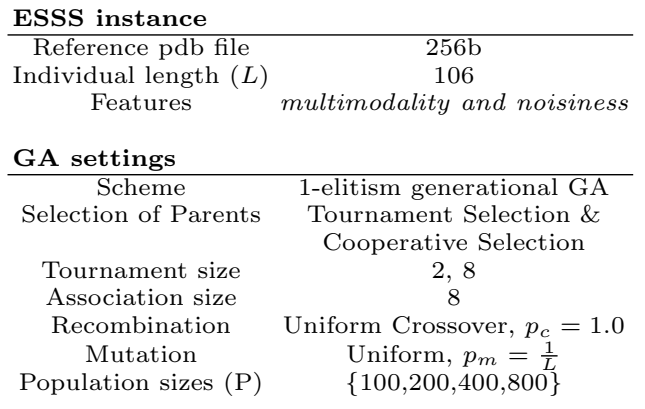

Table 1. Parameters of the experiments

\subsection{Analysis of results}

Miller and Goldberg [8] were the first in analyzing the relations between noisy functions and selection schemes. In general, noise has a disruptive effect on the convergence of a genetic algorithm, delaying the convergence rate and increasing the computational requirements. The common approach to counteract this effect is to increase the selection intensity. However, if the selection pressure is too high, the algorithm risks to prematurely converge to sub-optimal solutions. Noisy functions are therefore an attractive context to validate the properties of cooperative selection: the new selection scheme has been shown to increase the selection frequency of the fittest individuals (i.e. selection intensity) without paying an additional cost in diversity. 
Unlike other noisy problems (see e.g. [5] for a survey), ESSS does not include a true fitness function to recalibrate errors of the meta-model. Therefore, diversity is prioritized over quality in such a way that results can be validated a posteriori in the lab [2]. That leads to the notion of feasible region: a range of fitness values used as termination criteria. We assume the feasible region to be $[F(256 b, 256 b), F(4 J E A, 256 b)]=[463,520]$ for the instance under study.

Fig. 5 shows that cooperative selection $(a=8)$ yields better results than tournament selection $(t=2)$ while the genetic diversity is similarly preserved in both schemes as it was predicted in section 2.2 for analogue parametrizations. On the other hand, tournament selection converges faster than cooperative selection if the algorithms are equally parametrized $(a=8=t)$. However, a high value of tournament selection $(t=8)$ increases the selection pressure and the genetic diversity is significantly diminished.
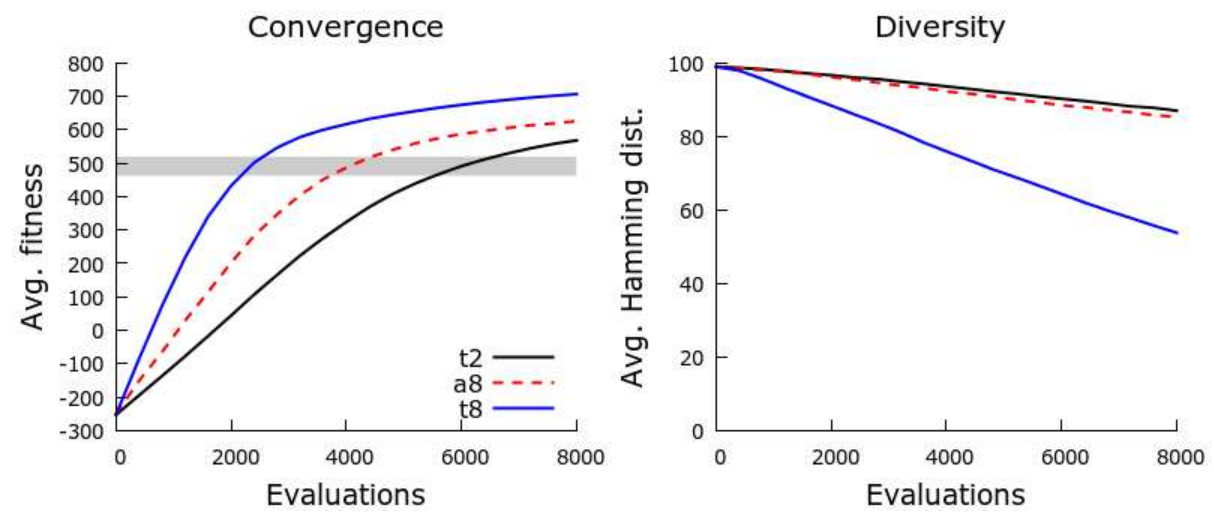

Fig. 5. Convergence of the average fitness in cooperative and tournament selection (left) and respective average hamming distance (right). The area in gray denotes the feasible region. Results are obtained over 30 independent runs for $P=400$.

In order to find best trade-offs between speed of convergence and preservation of diversity, we reproduce previous experiments for $P \in\{100,200,400,800\}$ with $\bar{F}=520$ set as the termination condition. Fig. 6 shows the results for cooperative and tournament selection where the upper-left corner is the optimal area. In all cases and independently of the population size, cooperative selection has better results regarding diversity and number of evaluations. That is, cooperative selection outperforms tournament selection. Although such results must be interpreted under the perspective of this study, the obtained conclusions should be easily generalized to further problems and optimization paradigms. 


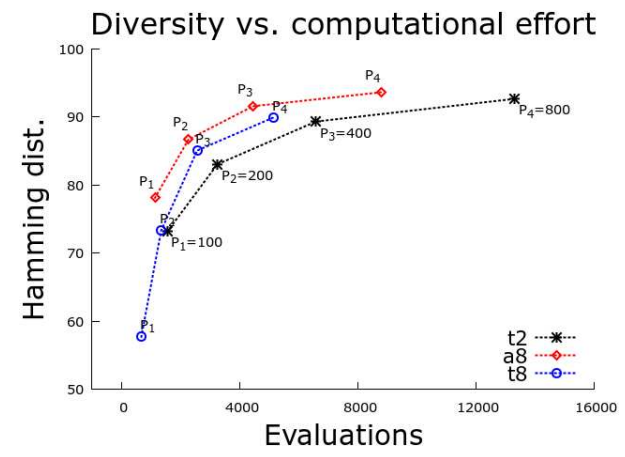

Fig. 6. Trade-off between diversity and computational effort for cooperative selection $\{a 8\}$ and tournament selection $\{t 2, t 8\}$.

\section{Conclusions and Future Works}

This paper analyzes a new selection scheme that we call cooperative selection in the context of genetic algorithms. The scheme is an extension of tournament selection that, by implementing an altruistic behavior in winners of the competition, is able to cope with two of the main problems of tournament selection: the "not-sampling" and "multi-selection" of individuals. On the one hand, the approach preferentially uses large association sizes, which neglects the probability of "not-sampling" an individual. On the other hand, the depreciation of the fitness in winners of the competition diminishes the "multi-selection" of the same elite of individuals.

We show that the new scheme preserves a higher genetic diversity than tournament selection when both schemes are equally parametrized. Additionally, cooperative selection casts fitter individuals when the loss of diversity is equal in both schemes. That allows the algorithm to converge faster to quality solutions while preserving the genetic diversity. The key to explain such a behavior is that the new scheme can select the same variety of individuals as in tournament selection but choosing preferentially fitter ones.

In order to validate such properties in a real problem, we formulate in this paper a new combinatorial problem for maximizing the similarity between proteins. The conducted experiments confirm previous conclusions: cooperative selection outperforms tournament selection for any setting of the parameters and is the best trade-off, maximizing genetic diversity and minimizing computational efforts.

As a future work, we plan to proceed with the mathematical modeling of the proposed selection scheme. Additionally, we also plan to investigate the adequacy of the approach in high-dimensional continuous optimization problems. 


\section{Acknowledgements}

This work has been funded by the UL-EvoPerf project. Fernandes wishes to thank FCT his Research Fellowship (SFRH/ BPD/66876/2009). This work was also supported by FCT PROJECT [PEst-OE/EEI/LA0009/2013].

\section{References}

1. Enrique Alba and Bernabé Dorronsoro. The exploration/exploitation tradeoff in dynamic cellular genetic algorithms. IEEE Trans. Evolutionary Computation, 9(2):126-142, 2005.

2. A.T. Brünger, P.D. Adams, G.M. Clore, W.L. DeLano, P. Gros, R.W. GrosseKunstleve, J. Jiang, J. Kuszewski, M. Nilges, N.S. Pannu, R.J. Read, L.M. Rice, T. Simonson, and G.L. Warren. Crystallography and NMR System: A New Software Suite for Macromolecular Structure Determination. Acta Crystallographica Section D, 54(5):905-921, Sep 1998.

3. A. E. Eiben, M. C. Schut, and A. R. De Wilde. Boosting genetic algorithms with self-adaptive selection. In In Proceedings of the IEEE Congress on Evolutionary Computation, pages 1584-1589, 2006.

4. Agoston E. Eiben and J. E. Smith. Introduction to Evolutionary Computing. SpringerVerlag, 2003.

5. Yaochu Jin and J. Branke. Evolutionary optimization in uncertain environments-a survey. Trans. Evol. Comp, 9(3):303-317, June 2005.

6. J.L.J. Laredo, B. Dorronsoro, C.M. Fernandes, J.J. Merelo, and P. Bouvry. Oversized populations and cooperative selection. In G. Nicosia and P. Pardalos, editors, Learning and Intelligent Optimization, pages 444-449. Springer Berlin Heidelberg, 2013.

7. J.L.J. Laredo, A.E. Eiben, M. van Steen, and J.J. Merelo. On the run-time dynamics of a peer-to-peer evolutionary algorithm. In Günter et al., editor, Parallel Problem Solving from Nature X, pages 236-245. Springer, 2008.

8. Brad L. Miller and David E. Goldberg. Genetic algorithms, selection schemes, and the varying effects of noise. Evol. Comput., 4(2):113-131, June 1996.

9. Riccardo Poli. Tournament selection, iterated coupon-collection problem, and backward-chaining evolutionary algorithms. In Wright et al., editor, Foundations of Genetic Algorithms, volume 3469 of Lecture Notes in Computer Science, pages 132-155. Springer Berlin Heidelberg, 2005.

10. B. Rost and C. Sander. Combining evolutionary information and neural networks to predict protein secondary structure. Proteins, 19(1):55-72, 1994.

11. J. David Schaffer and Larry J. Eshelman. On crossover as an evolutionarily viable strategy. In Richard K. Belew and Lashon B. Booker, editors, ICGA, pages 61-68. Morgan Kaufmann, 1991.

12. Artem Sokolov and Darrell Whitley. Unbiased tournament selection. In Proceedings of the 2005 conference on Genetic and evolutionary computation, GECCO '05, pages 1131-1138, New York, NY, USA, 2005. ACM.

13. Huayang Xie and Mengjie Zhang. Impacts of sampling strategies in tournament selection for genetic programming. Soft Comput., 16(4):615-633, April 2012.

14. Huayang Xie, Mengjie Zhang, and Peter Andreae. Another investigation on tournament selection: modelling and visualisation. In Proceedings of the 9th annual conference on Genetic and evolutionary computation, GECCO '07, pages 14681475, New York, NY, USA, 2007. ACM. 